\title{
Visual analysis of controversy in user-generated encyclopedias
}

\author{
Ulrik Brandes ${ }^{1}$ \\ Jürgen Lerner ${ }^{1}$ \\ ${ }^{1}$ Department of Computer \& Information \\ Science, University of Konstanz, Konstanz, \\ Germany

\section{Correspondence:} \\ Jürgen Lerner, \\ Department of Computer and \\ Information Science, University of \\ Konstanz, Box D 67, 78457 Konstanz, Germany. \\ Tel: +497531 884436; \\ Fax: +497531883577; \\ E-mail: lerner@inf.uni-konstanz.de
}

\begin{abstract}
Wikipedia is a large and rapidly growing Web-based collaborative authoring environment, where anyone on the Internet can create, modify, and delete pages about encyclopedic topics. A remarkable property of some Wikipedia pages is that they are written by up to thousands of authors who may have contradicting opinions. In this paper, we show that a visual analysis of the 'who revises whom'-network gives deep insight into controversies. We propose a set of analysis and visualization techniques that reveal the dominant authors of a page, the roles they play, and the alters they confront. Thereby we provide tools to understand how Wikipedia authors collaborate in the presence of controversy.

Information Visualization (2008) 7, 34-48. doi: I0. I 057/palgrave.ivs.9500 I7I
\end{abstract}

Keywords: Wikipedia; social network analysis; controversy

\section{Introduction}

Recently, the World Wide Web (WWW) has witnessed a shift from websites supplied by traditional information providers like universities or companies to sites where every user can not only read but also modify content. A remarkable example of such sites is the user-generated online encyclopedia Wikipedia which allows every user (even anonymously) to create, modify, and delete pages about encyclopedic topics. This approach - which is so entirely different from traditional encyclopedia-writing by domain experts and supervised by editors - seemed to be destined to fail from the beginning. Not only could users (ignorantly or maliciously) introduce inaccurate information but also could delete previously written good articles, thereby making every progress impossible. Despite these concerns, Wikipedia turned out to produce much better articles than expected. A study carried out by Nature in 2005 suggests that the accuracy of Wikipedia articles about scientific topics comes close to the accuracy of their counterparts in the Encyclopædia Britannica. ${ }^{2}$ Viégas et al..$^{3,4}$ observed that antisocial behavior like vandalism (e.g., deletion of whole pages or insertion of vulgarities) is often repaired within minutes. Another indicator of Wikipedia's success is simply its ever-increasing popularity: at the end of 2006, Wikipedia has more than five million articles - about 1.5 million alone in the English Wikipedia and grows by several thousand articles per day (http://stats.wikimedia.org/). Furthermore, Wikipedia ranges among the top 20 in Alexa's most visited sites (http://www.alexa.com/).

In this paper, we are interested in how do Wikipedia authors collaborate when writing about controversial topics (such as abortion, gun rights $v s$ gun control), delicate historic events, or persons who are highly important in politics. Such pages have often been revised up to tens of thousands of times by several thousand authors who, arguably, not all share the same opinion on the particular topic. Although Wikipedia policies ${ }^{5}$ 


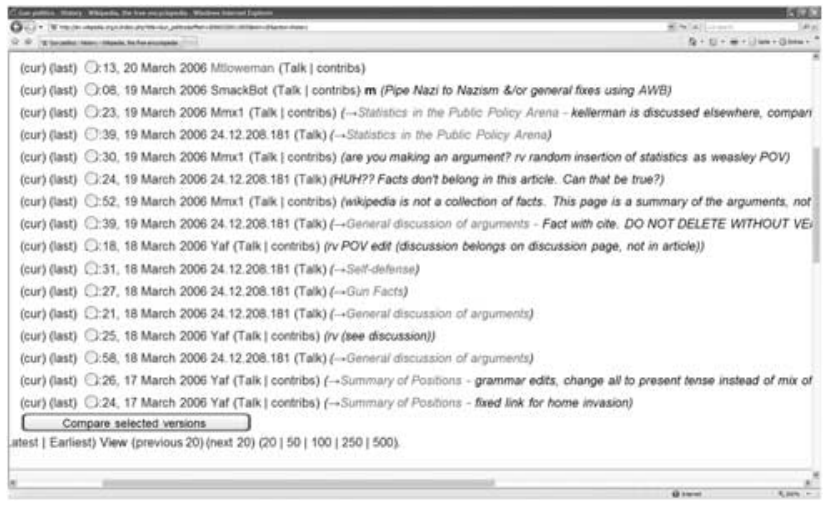

Figure 1 Small part of the revision history of the page Gun politics. This page has 1101 revisions in the November 2006 database dump.

urge authors to take a neutral point of view and to provide only facts rather than opinions, controversies are nevertheless reflected in some pages. Since some facts appear to support more a certain opinion and reject or discredit the other, it is fiercely fought over whether such facts should be mentioned and how could balance be established.

We do not see it as a fundamental drawback of Wikipedia that controversies are reflected in the development of (some of) its pages. Different opinions simply exist in society and, since Wikipedia is 'the free encyclopedia that anyone can edit,' it is a good mirror of such controversies. However, this gives rise to several important questions: First of all, to assess the neutrality of a given controversial page, it is crucial (and very informative) to know about ongoing and past disputes and about beliefs and opinions of the various authors. Even more important is to understand in general the social process of content-generation in Wikipedia. Concrete questions include whether controversial pages converge at all or whether they are destined to perpetual editing and, if they converge, is their content balanced or determined by opinion groups. Furthermore, what are the roles that Wikipedia authors typically play when arguing for or against specific statements in the page.

Support to answer these questions comes from Wikipedia itself which makes available not only the current content of a page but also its complete history. The analyst is thereby enabled to see all past versions and time, content, comment, and author of the various edits (see e.g., Figure 1). Needless to say that the typical size of the revision history of a disputed page calls for automated visual and analytic support to get insight into the page's development and author community.

In this paper, we show that a visual analysis of the 'who revises whom'-network gives deep insight into the authorcommunity behind a controversial page. We provide a set of analysis and visualization techniques that reveal the dominant authors, the roles they play, and the alters they confront.

The rest of this paper is organized as follows. Our contributions in relation to previous work are explained in the next section. In the subsequent section we define the revision network. The fourth section introduces several meaningful author properties and how they are visually represented and the next section presents some illustrating findings on particular pages.

\section{Related work and contributions}

Web 2.0 is a common term for denoting those sites of the WWW where Internet users are not just readers but can actively participate. Specific forms include blogs, wikis, podcasting, file sharing, and social networking sites (see, e.g., Kolbitsch and Maurer ${ }^{6}$ for an overview). In this paper, we analyze the author community of wikis, that is, Web-based collaborative authoring environments where anyone on the Internet can create, edit, and delete pages. The term wiki was coined by Ward Cunningham, who launched the first wiki in $1995 .^{7}$ Wikipedia, which is currently the largest wiki, has been established in 2001 to collectively create an encyclopedia. Maybe due to its size, popularity, and relevance for understanding new forms of collective knowledge creation, Wikipedia receives increasing interest in research. For instance, Wikipedia's growth rate, information quality, or edit histories have been analyzed. , $^{4,8-10}$ Other papers (e.g., Gabrilovich and Markovitch ${ }^{11}$ and Strube and Ponzetto ${ }^{12}$ ) use the collection of Wikipedia articles to improve machine learning techniques for text categorization and detection of semantic relatedness of terms.

It has been widely recognized that user-generated content is also a rich source for user opinions. Some papers (e.g., Chen et al., ${ }^{13}$ Glance et al., ${ }^{14}$ Liu et al. ${ }^{15}$ and Nigam and Hurst ${ }^{16}$ ) apply natural language processing (NLP) to determine users' sentiments about positive or negative aspects of commercial products. Agrawal et al. ${ }^{17}$ argued that 'links carry less noisy information than text' and applied a network analysis approach to divide newsgroup authors into two opposite camps: those that have a positive opinion on a certain topic and those that have a negative opinion. They completely ignored the content of postings and used only the 'responded-to' relationship between authors. It is argued (and validated) that people respond more frequently to a message when they disagree than when they agree. Thus, partitioning the network into two groups such that most links are between the groups will reveal the opposing camps. Note that previous work on user opinions ${ }^{13-17}$ assumes the existence of only two poles of opinion (positive and negative), which is certainly a restriction to generality. However, research about multipolar conflicts (i.e., situations where there are more than two camps that are mutually in opposition) can be found in political science, for example Rosecrance. ${ }^{18}$ 
Our work here is based on the idea from Agrawal et al. ${ }^{17}$ that controversy is reflected in the reply behavior (revision behavior in our case) of authors but achieves several improvements. Instead of the strict partition of authors into opinion groups, we propose a visual analytics approach that can deal with more complex and more realistic controversy structures and in addition reveals authors' involvement and roles.

Independently, Kittur et al. ${ }^{19,20}$ applied a similar idea by building the RevertGraph to analyze disagreement among authors. Our proposal of the revision network can encode conflicts in more general situations, since using only reverts 'cannot detect conflicts between users who were not involved in reverts' Kittur, ${ }^{19}$ [p. 460]. Furthermore, in addition to different opinion groups, our method reveals several author characteristics. Last but not least, the spectral layout method outlined in Section 'What position do they take?' seems to be preferable to the force-directed method from Kittur et al. ${ }^{19}$ and Suh et al. ${ }^{20}$ since it optimizes a well-defined criterion function, cannot be stuck in local minima, and is quite robust to noise (compare Brandes et al. ${ }^{21}$ ). Note that Kittur et al. provide additional results in different directions by analyzing the global cost of coordination and learning models to predict whether an article is controversial.

Viégas et al. ${ }^{3,4}$ proposed a history flow approach for the visual analysis of the page history. The history flow diagrams show the development of the content of a page over time and are therefore orthogonal to our work since we analyze the page's author community.

The determination of the authors' positions developed in Section 'What position do they take?' is a generalization of the method that we proposed for the analysis of political conflicts. ${ }^{21}$ The method from the current paper can deal with more general conflict structures (e.g., multipolar conflicts). Furthermore, we make several visual and analytical enhancements that have been necessary to represent well the complex interaction structure between Wikipedia authors.

Concrete contributions of our paper include the following. First, the definition of the revision network is a simple, efficient, and language-independent way to represent controversies among Wikipedia authors. Note that this approach can be applied to Wikipedia articles in any language without the need for adapting NLP algorithms. This is a significant advantage since for most languages, text processing algorithms are not so highly developed as for English. Second, we define a set of author characteristics or properties that give deep insight into the overall structure of the community as well as into individual authors' roles. Third, we develop visualization techniques to show the author characteristics simultaneously in a simple and easy to understand picture. Last but not least, several case studies of controversial pages have a value on their own in revealing some typical author roles and patterns of confrontation.
It is important to note that our analysis cannot and does not attempt to determine which opinion is more acceptable.

\section{Revision network}

The definition of the 'who-revises-whom'-network (in short revision network) is a crucial step to develop an efficient and robust method for analyzing interaction among Wikipedia authors. In contrast, approaches based on NLP would not only have to solve the difficult task of automatically understand natural language (compare Agrawal et al. ${ }^{17}$ ) but would also have to deal with much larger file sizes (see Section 'Input data'). We describe the input data in the subsection below before defining the revision network in the next subsection.

\section{Input data}

Wikipedia makes its complete database (containing all versions of every article since its initial creation) available in XML-format. ${ }^{22}$ The files containing the complete history of all pages can be extremely large. For instance, the complete dump for the English Wikipedia unpacks to more than 600 gigabytes (GB). Wikipedia makes also available so-called stub-files. These files contain metadata about every revision but not the text (see Figure 2 for a small portion) and are still quite large. For the present study we used the stub-file for the English Wikipedia (which is the largest one) from the 20061130 dump with a size of $23 \mathrm{~GB}$. (Note that this dump includes some revisions from December 2006, since it takes several days to create it.) The number of revisions (edits) of a page and the number of authors that made at least one revisions can also be quite large. The most-revised page in the English Wikipedia is George W. Bush having 33,086 revisions and 10,167 different authors (registered or anonymous). Parsing the XML-document has been done using a Java implementation of the event-based SAX interfaces $^{23}$ which proved to be very efficient for parsing such huge files. Constructing the whole document tree, as this is normally done by DOM parsers, ${ }^{24}$ would simply be impossible (at least very inefficient and/or requiring uncommonly huge memory), given the file sizes.

To abstract from the particular format we define a revision or edit to be a tuple of the form

$r=($ page, time, author, comment, revert),

where page is a text-string denoting the page-title, time contains the exact timestamp of the revision (given by the second), author is a real user name if the contributor of the revision has been logged in or an IP-address if the revision has been done anonymously, comment is free text explaining what has been done or why this revision has been necessary (often authors have kind of a discussion in consecutive comments, compare Figure 2), and revert is a Boolean flag labeling the revision. (A revert is a specific 


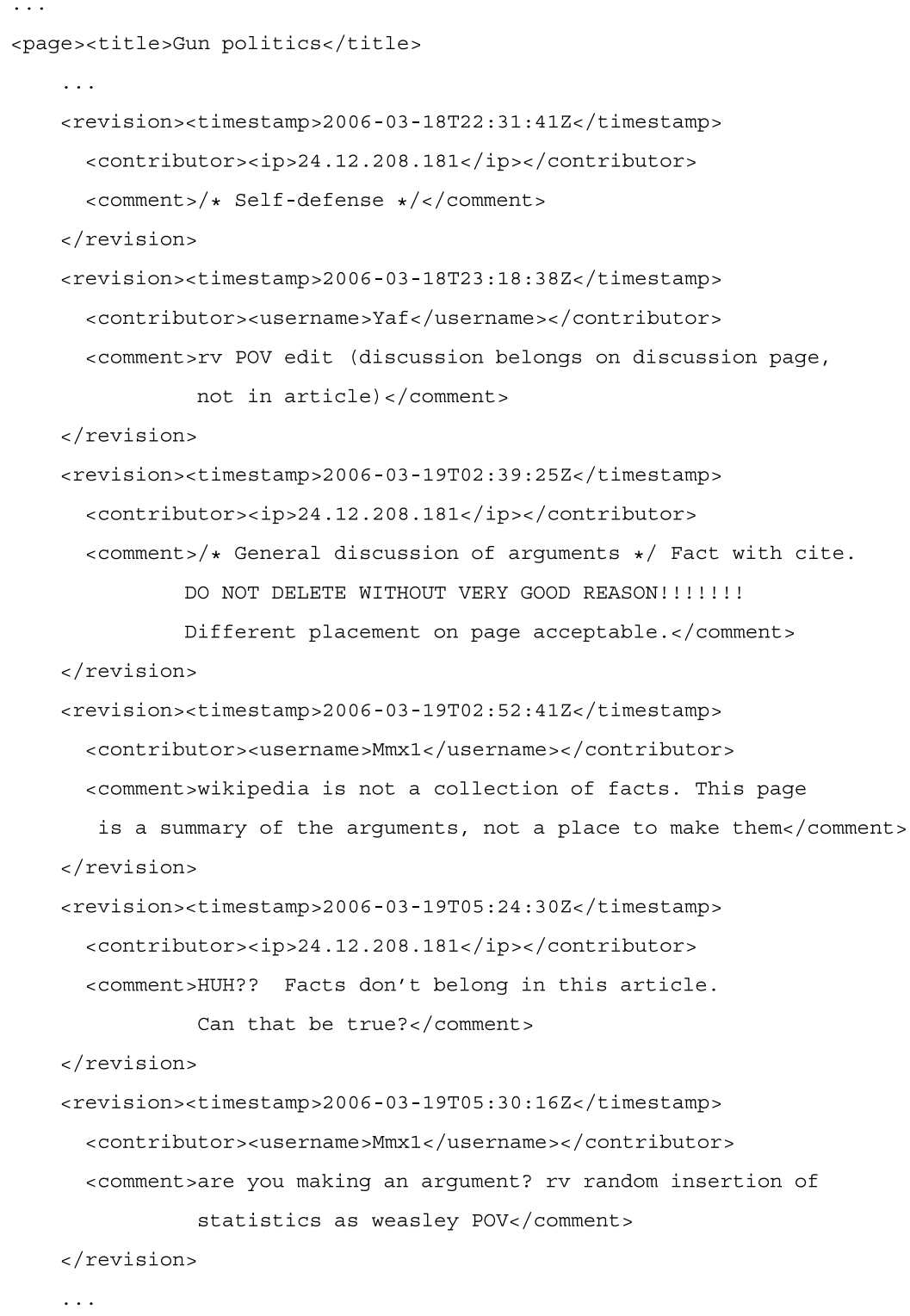

Figure 2 Six consecutive revisions of the page Gun politics in XML format. (The corresponding HTML-view is part of Figure 1.)

edit where the author sets back the page content to an earlier version.)

\section{Network construction}

Given a sequence $R=\left(r_{1}, \ldots, r_{N}\right)$ of revisions on the same page, which is ordered by increasing timestamps, the associated revision network is a directed, weighted graph $G=$ $(V, E, \omega)$ defined as follows (also compare Figure 3 ).

- $V$ is the set of authors that performed a revision in $R$.

- $E \subseteq V \times V$ is the set of revision edges. For two different authors $u, v \in V$ the edge $(u, v) \in E$ is introduced if there are two consecutive revisions $r_{i}, r_{i+1} \in R$ such that $u$ is the author of $r_{i+1}$ and $v$ the author of $r_{i}$. An edge $(u, v)$ can be read as ' $u$ revises changes made by $v$ '.

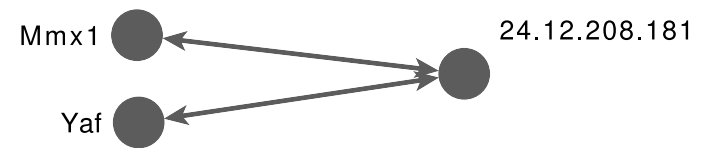

Figure 3 Revision network arising from the six revisions shown in Figure 2. Both edges go in both directions but edges from the left to the right have higher weights since the corresponding revisions are performed faster, compare (1).

- The function $\omega: R \rightarrow \mathbb{R}$ assigns weights to edges. For an edge $(u, v)$ the weight $\omega(u, v)$ indicates how 'urgent' $u$ considers it to revise the changes made by $v$ (see more detailed explanation below). 
Before explaining how the edge weights are defined we will briefly discuss the meaningfulness of the revision network. Edges with high weight are interpreted later as disagreements between the connected authors. To see how the edge weights have to be defined to achieve this goal, assume that there are two (fictitious) authors Alice and Bob connected by an edge. If Alice makes only once a revision immediately after Bob, then this may or may not indicate that she disagrees with his edits. If, on the other hand, it is the case that Alice revises dozens of times Bob's revisions (and especially if these revisions happen very fast, e.g., within an hour or even within minutes), then it becomes very likely that she does not at all agree with his edits. It turns out later that there are indeed such pairs of authors on some highly controversial pages. To summarize these considerations, we assume that dominant revision patterns are meaningful but that not too much confidence should be put on single revisions. This simply means that the revision network has a typical characteristic of social network data, namely that of being noisy, and that it should only be analyzed/visualized with robust methods. In a sense, the same considerations would apply to the construction of 'quotation links' for the analysis of newsgroups. ${ }^{17}$ There it has been claimed that 'it is more likely that the quotation is made by a person challenging or rebutting it rather than by someone supporting it' Agrawal et al. ${ }^{17}$ [p. 529]. Of course not every single quotation is necessarily antagonistic, but a huge number is likely to indicate disagreement.

Thus, to define edge weights such that they are likely to indicate the magnitude of disagreement, fast revisions are assigned higher weights and weights of several revisions between the same authors are added up. So, let $r_{i}, r_{i+1}$ be two consecutive revisions on the same page where $u$ is the author of $r_{i+1}$ and $v$ the author of $r_{i}$. Let $t_{i}$ and $t_{i+1}$ denote the timestamps of $r_{i}$ and $r_{i+1}$ respectively, $\Delta=t_{i+1}-t_{i}$ the time difference between the two revisions, and $\Delta_{\max }$ a maximum time limit when a revision is still considered as a disagreement. Then, the weight of the edge $(u, v)$ is defined to be

$\omega(u, v)= \begin{cases}-\Delta / \Delta_{\max }+1 & \text { if } \Delta \leqslant \Delta_{\max } \\ 0 & \text { else. }\end{cases}$

If there are more pairs of consecutive revisions where $u$ revises $v$, then the edge weights of $(u, v)$ are summed up.

In the examples we defined the time limit $\Delta_{\max }$ to be equal to the average revision time. If a revision occurs at about the average time, it becomes more unlikely that it is meant as a disagreement. On the other hand if the revision occurs much faster than the average time, the probability increases that it is indeed a correction of the previous edit. It is reasonable to count revisions more heavily if they are reverts since this indicates that the reverting author considers the previous edit as obsolete or even harmful. An even more sophisticated construction of revision edges could be achieved by taking into account the comments made by authors. Since comments are free text and not standardized this would involve NLP and will not be considered in this paper.

Sometimes several Wikipedia pages have strongly related topics (see, e.g., Section 'Gun politics') and then often largely overlapping sets of authors. In these situations, it is appropriate to combine the associated revision networks by taking the union of their author sets and adding up edge weights.

\section{Visual analysis of the revision network}

In this section, we define a series of characteristics of the revision network and its actors (the Wikipedia authors) and how they are visually represented. These characteristics include for all authors their position (i.e., which other authors do they confront), their involvement in controversy, an indicator telling whether they are mostly revisors or mostly being revised, and an indicator telling whether their edit behavior is rather constant over time (so that they showed sustained interest in the page) or highly concentrated on small time periods. See Figure 4 for an image showing these and a few other properties. Technically most involved is the determination of the authors' positions. We will treat this issue in the next subsection. Graphical representation of this and other indicators is explained and illustrated in Section 'Visual representation of author properties'. In Section 'Filtering', two possibilities to prune the revision network and to detect relevant substructures are examined.

\section{What position do they take?}

The position of a particular author should express which other authors she confronts. Confrontation is reflected in the revision edges: if two authors take different positions they disagree with the edits of the other and therefore will frequently revise each other. (Asymmetry of edges is ignored here but will be used later to determine the authors' roles.) Thus, if two authors $u$ and $v$ are connected by a revision edge of large weight, then we want to draw $u$ and $v$ on opposite sides of the image. The difficulty lies in the fact that we have to draw not only two authors but also the whole network such that all confronting pairs are simultaneously as far from each other as possible (compare Figure 5). This objective (which contrasts to most objective functions for graph drawing that traditionally want to keep edge lengths as short as possible ${ }^{25}$ ) is of course due to the negative interpretation of the revision edges. The good news is that this problem is efficiently solvable, as will be derived next.

Let $G=(V, E, \omega)$ be a revision network with author set $V$ of cardinality $n=|V|$. We associate with $G$ its symmetric adjacency matrix $A=\left(a_{u v}\right)$ with rows and columns indexed by $V$ and entries $a_{u v}=\omega(u, v)+\omega(v, u)$ corresponding to the sum of the weights of the two directed edges between the two endpoints (if an edge is not present, the weight is simply equal to zero). We want to draw the conflict network in two-dimensional space. Thus, the positions of 


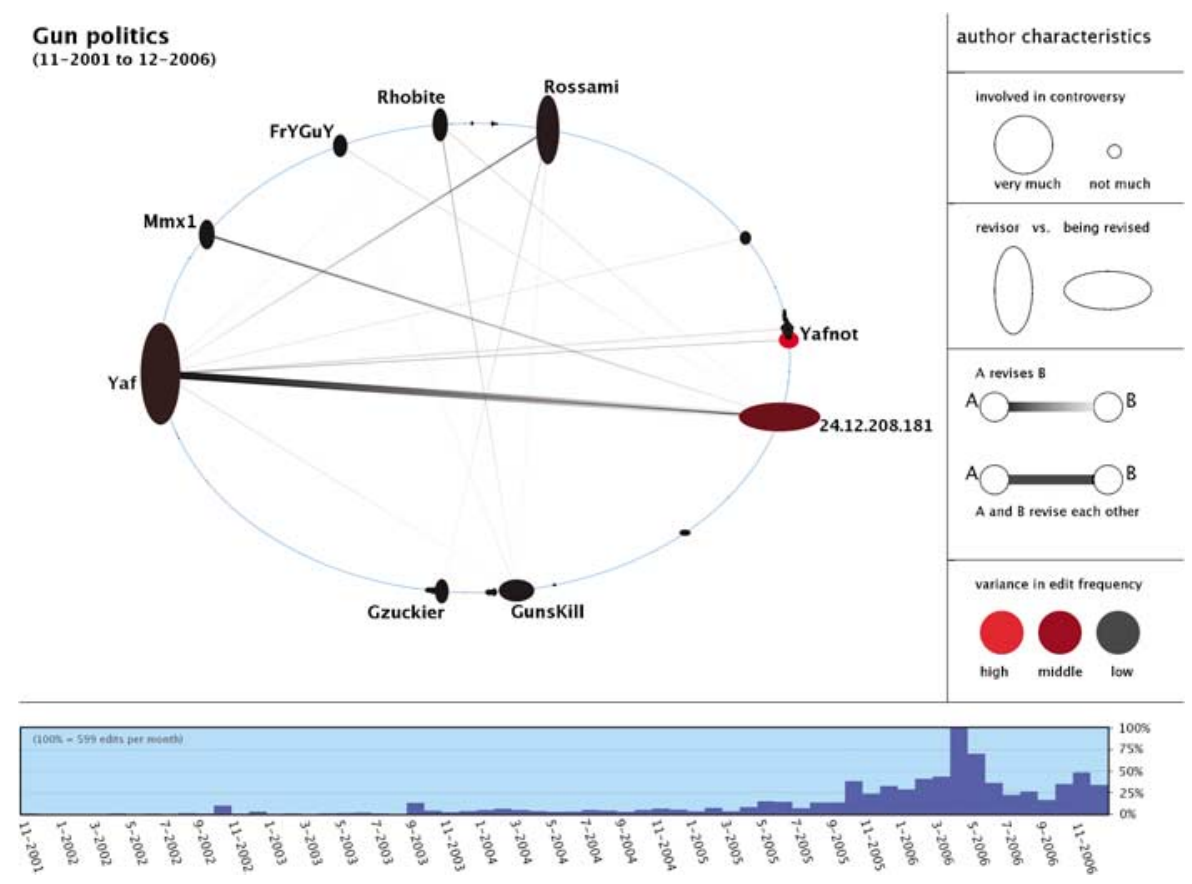

Figure 4 Example visualization of a revision network (determined from Gun politics and related pages). Nodes represent the different authors. If two authors are on opposite sides they strongly revise each other. Other characteristics are represented as described in the legend on the right-hand side (also see Section 'Visual representation of author properties'). The diagram at the bottom shows the total number of edits per month. For more on this particular network, see Section 'Gun politics'.

all $n$ authors are represented by two vectors $x, y \in \mathbb{R}^{n}$. If for two authors $u$ and $v$ the entry $a_{u v}$ in the adjacency matrix is large (i.e., if they frequently revise each other), then they are well-represented by the coordinate vector $x$ if the entry $x_{u}$ is (say) strongly negative and the entry $x_{v}$ strongly positive. Then, the value $x_{u} a_{u v} x_{v}$ is negative and has quite large absolute value. Summing this up over all pairs of authors, $x$ is determined to minimize the objective function

$\Phi_{A}(x)=\sum_{u, v \in V} x_{u} a_{u v} x_{v}=x^{\mathrm{T}} A x$

under the condition that $x$ must have unit length (to keep the drawing to the screen size). It follows from an alternative description of the eigenvalues of a matrix that this term is minimized if and only if $x$ is equal to the eigenvector of $A$ associated to the smallest eigenvalue $\lambda_{\text {min }}$ (see, e.g., Golub and van Loan ${ }^{26}$ ). The second coordinate vector $y$ is chosen to minimize $\Phi_{A}(y)$ under the condition that $y$ is normalized and orthogonal to $x$. This is solved by taking for $y$ the eigenvector of $A$ associated to the second smallest eigenvalue $\lambda_{\text {min }}^{\prime}$.

The coordinate vectors derived so far would already represent well some pure conflict patterns as in Figure 5 (middle) and (right). However, real data is normally not so balanced. For instance, it might be the case that in Figure 5 (middle) one side of the triangle consists only of very weak edges so that it approaches a bipolar conflict
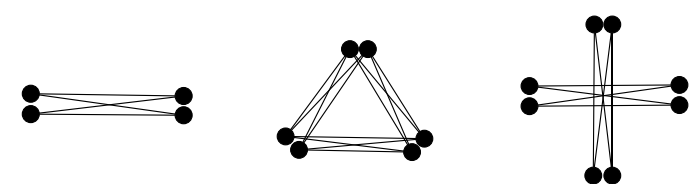

Figure 5 Sample of pure conflict patterns. Bipolar conflict (left), 3-polar conflict (middle), and two independent bipolar conflicts (right). Actors that are in conflict are drawn as far from each other as possible. Conflicts in real data are often a mixture of these types.
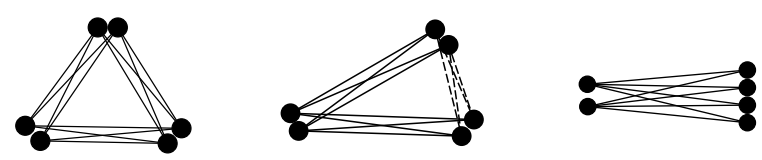

Figure 6 Smooth transformation from pure 3-polar conflict (left) to bipolar conflict (right). The dashed edges of the intermediate graph (middle) are assumed to have lower weight.

(compare Figure 6). To achieve a smooth transformation between different conflict patterns, we scale $y$ with the ratio between the two minimal eigenvalues $\lambda_{\text {min }}^{\prime} / \lambda_{\text {min }}$. The derivation why this rescaling interpolates between different conflict patterns is deferred to the next section 'Derivation of the scaling factor'. Note that a justification 


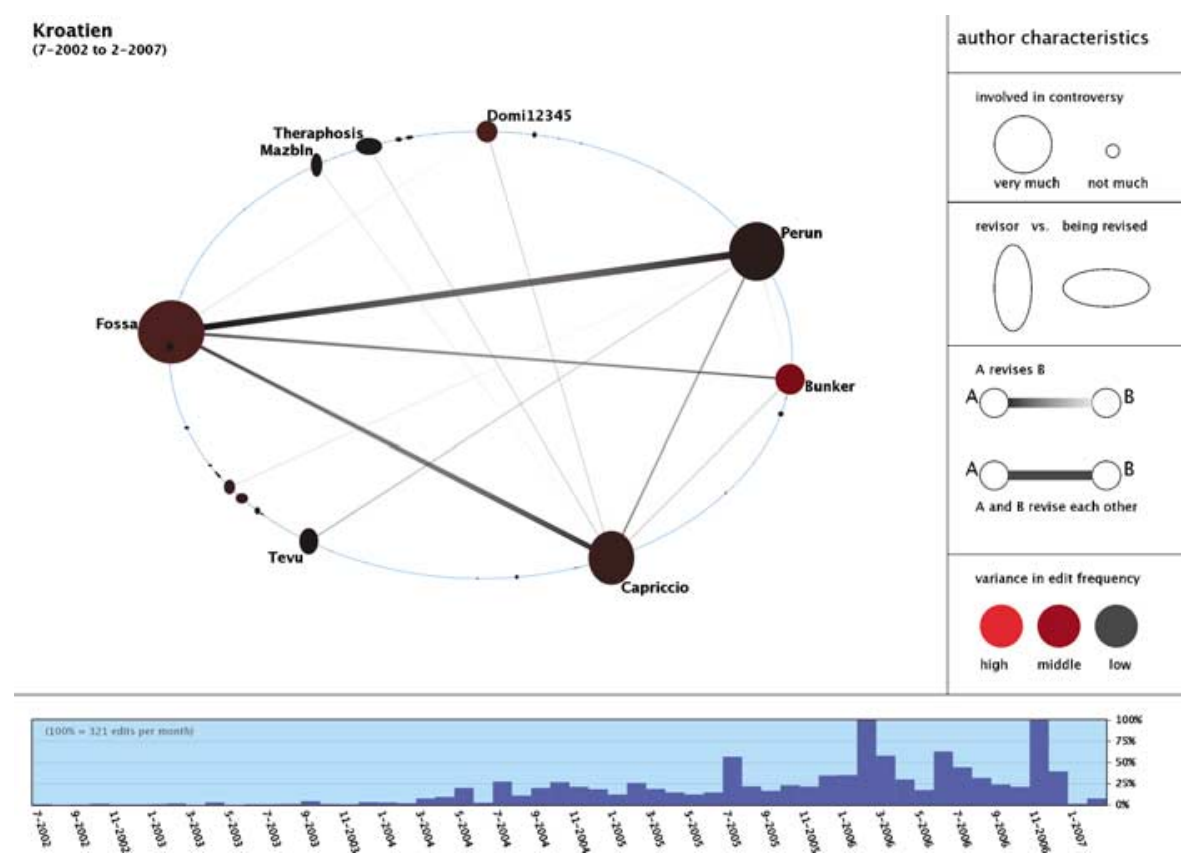

Figure 7 Revision network related to the page Kroatien (Croatia) in the German Wikipedia. The three most involved authors are mutually in conflict and are displayed as a triangle. Note that the edge weights between these three authors vary and less connected users are drawn closer together, compare Section 'Derivation of the scaling factor'.

is also provided by the examples shown in this article (compare Figure 7).

The absolute values of the two coordinates of an author $v$ are a measure of how much $v$ is involved in controversy, since they indicate how strongly $v$ is connected to others via revision edges.

Putting this together, we get the following algorithm for determining the authors' positions and involvement, which takes as input the symmetric adjacency matrix $A$ of the revision network.

1. Compute the smallest and second smallest eigenvalue $\lambda_{\min }$ and $\lambda_{\text {min }}^{\prime}$ of $A$ and the associated (normalized and orthogonal) eigenvectors $x$ and $y$.

2. Set $s=\lambda_{\min }^{\prime} / \lambda_{\min }$ as the network's skewness and define for an author $v$ its position $p(v)=\left(p_{1}(v), p_{2}(v)\right)=\left(x_{v}, s y_{v}\right) \in$ $\mathbb{R}^{2}$ and its involvement $i(v)=\sqrt{p_{1}(v)^{2}+p_{2}(v)^{2}}$.

Efficient computation of the extremal eigenvalues and vectors is possible, for example, with the so-called orthogonal iteration, which can also exploit sparsity of the network (see Golub and van Loan. ${ }^{26}$ )

Note that, although our layout method seems to be similar to multidimensional scaling (MDS) on a distance matrix, it enjoys a further desirable property: MDS would try to achieve distance zero for all authors that are not connected, whereas our method requires in addition that authors must confront (approximately) the same others to be placed at the same position. Thereby, independent conflicts (as in Figure 5 (right)) can be recognized as such in the final drawing.
Derivation of the scaling factor As it is mentioned above, we propose to scale the eigenvector $y$ that is associated to the second smallest eigenvalue with the ratio between the two minimal eigenvalues $\lambda_{\text {min }}^{\prime} / \lambda_{\text {min }}$. Here we want to give a heuristic derivation of this particular choice, illustrated on hypothetical networks that exhibit certain pure conflict structures.

As a simple example, assume that the revision network has a structure similar to that in Figure 5 (right), but where the disagreement edges on the vertical axis have a smaller weight than those on the horizontal axis. More precisely, assume that the authors of the revision network are partitioned into four classes $C_{1}, \ldots, C_{4}$, where each class consists of $r$ authors. Furthermore, assume that every author in class $C_{1}$ is connected to every author in class $C_{2}$ by an edge of weight $w$ (the horizontal conflict), that every author in class $C_{3}$ is connected to every author in class $C_{4}$ by an edge of weight $\delta w$, for a $\delta$ between zero and one, (the vertical conflict), and that the network has no other edges. It is easy to show that in this case the two minimal eigenvalues are $\lambda_{\min }=-r w$ and $\lambda_{\text {min }}^{\prime}=-\delta r w$. Furthermore, the eigenvector $x$ associated to $\lambda_{\min }$ assigns (modulo normalization) the value 1 to every author in class $C_{1}$, value -1 to every author in class $C_{2}$ and zero to authors in classes three and four. Likewise, the eigenvector $y$ associated to $\lambda_{\min }$ assigns (modulo normalization) the value 1 to every author in class $C_{3}$, value -1 to every author in class $C_{4}$ and zero to authors in classes one and two. By scaling $y$ with the factor $\lambda_{\text {min }}^{\prime} / \lambda_{\min }=\delta$ and using $x$ and $\delta y$ as coordinates we obtain a network visualization similar to Figure 5 (right) but where the authors 


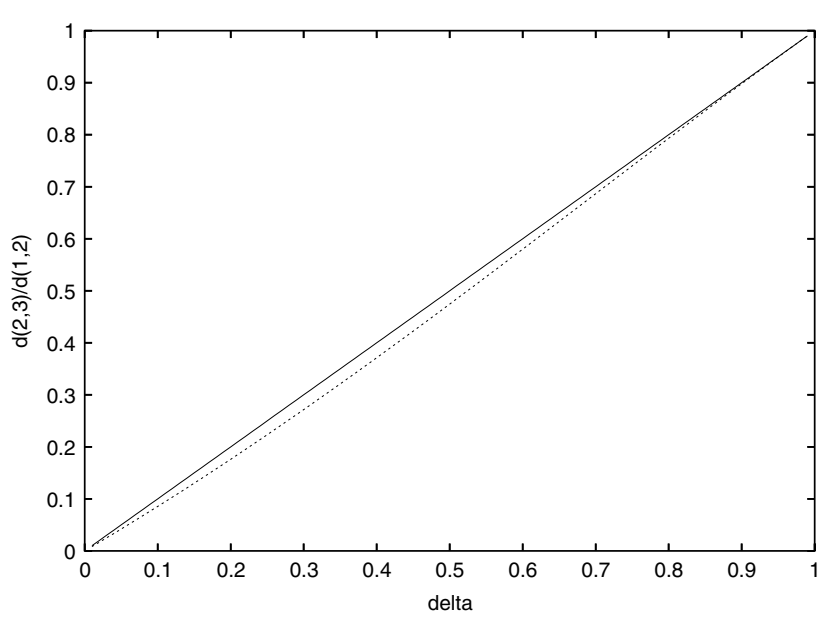

Figure 8 The dashed line shows the computed ratio $d(2,3) / d(1,2)$ for varying $\delta$. The straight line is the plot of the function $y=x$.

in classes $C_{3}$ and $C_{4}$ (the vertical conflict) are drawn at $\delta$ times the distance between authors in classes $C_{1}$ and $C_{2}$ (the horizontal conflict). Since the former pairs of authors disagree less (by a $\delta$ factor) than the latter, this layout represents the data quite well. From a different angle, the layout satisfies the property that the edge length between conflicting authors is proportional to the weight of the edges connecting them.

Likewise, we can show by simulation that this property (almost) holds for three-polar conflict structures and hence that the proposed scaling interpolates well between three-polar and bipolar conflicts, as illustrated in Figure 6 . For the simulation setup, assume that the set of authors is partitioned into three classes $C_{1}, \ldots, C_{3}$, each of size $r$, such that each author is connected by disagreement edges to all authors in the two other classes but not to authors in her own class. Assume further that the edges between classes $C_{1}$ and $C_{2}$ as well as the edges between classes $C_{1}$ and $C_{3}$ have a positive weight $w$, while the edges between classes $C_{2}$ and $C_{3}$ have a lower weight $\delta w$ for a $\delta$ between zero and one. By letting $\delta$ vary from one to zero we get a smooth interpolation from a balanced three-polar to a bipolar conflict structure and we want to show that the computed positions reflect this transformation. It is easy to show that authors in the same class are assigned the same position and authors in different classes are assigned different positions. Let $d(i, j), i, j=$ $1,2,3$ denote the distance between an author in class $C_{i}$ and an author in class $C_{j}$. Our goal is to show that it (almost) holds that $d(2,3)=\delta d(1,2)=\delta d(1,3)$, that is, that the classes $C_{2}$ and $C_{3}$ are by a $\delta$-factor closer to each other than class $C_{1}$ to $C_{2}$ or to $C_{3}$ (in particular, if $\delta$ varies from one to zero, then the classes $C_{2}$ and $C_{3}$ get closer until we display the structure of a pure bipolar conflict). This equation does not hold exactly but, as Figure 8 shows, the ratio $d(2,3) / d(1,2)$ is almost equal to $\delta$, and hence, the computed positions follow the network structure reasonably close.

\section{Visual representation of author properties}

Next we define several additional characteristics of the authors and explain how these (and the previously defined position and involvement) are graphically represented.

Position and involvement Let $\left(p_{1}(v), p_{2}(v)\right)$ be the position of an author $v$ and $i(v)$ her involvement as defined in the previous subsection. The position coordinates $\left(p_{1}(v), p_{2}(v)\right)$ could be directly used for drawing authors in two-dimensional space. However, when doing so, many of the not-so-important authors would be placed near the center of the drawing, making it hard to recognize their positions (compare Figure 9 with Figure 10). To overcome this we normalize the positions to draw authors on an ellipse: Let $r_{1}$ be the horizontal half axis (value determined by the size of the image) and $r_{2}=s r_{1}$ the vertical half axis. We draw author $v$ at the coordinates $\left(r_{1} \cdot p_{1}(v) / i(v), r_{2} p_{2}(v) / i(v)\right)$. Normalizing author positions to an ellipse rather than a circle has the advantage that we can still distinguish between the dominant conflict (shown horizontally) and secondary conflict (shown vertically) if their magnitude is different. Compare, for example, Figure 11, where the dominant conflict is much stronger than the secondary conflict, and 11 where the two major conflicts are of similar magnitude.

The area size of the node representing $v$ is proportional to the involvement $i(v)$ (shape will be explained in the subsection below). Thus, even after the normalization of positions it is still possible to distinguish between different involvement of authors.

We draw the usernames (or IP-addresses in case of anonymous contributors) of the most involved authors as node labels. Printing all usernames would produce clutter, considering that the revision networks can have several thousand authors.

Revisor vs being revised The out-degree $d^{+}(v)=\sum_{(v, u) \in E}$ $\omega(v, u)$ of an author $v$ indicates how strongly she revises others and is called her degree as a revisor, the indegree $d^{-}(v)=\sum_{(u, v) \in E} \omega(u, v)$ indicates how strongly she is revised by others and is called her degree of being revised. We draw $v$ as an ellipse with height/width-ratio proportional to $d^{+}(v) / d^{-}(v)$, while keeping the area size proportional to the involvement $i(v)$. (However, to avoid very thin ellipses we bound the aspect ratio.)

The distinction between revisors (high and narrow) and those that are revised (wide and flat) is a very interesting one: Those who are mostly revisors seem to be quite satisfied with a page and react only to revise changes made by others. On the other hand, those that are mostly revised attempt to initialize changes to a page that are not approved by the revisors and therefore corrected 


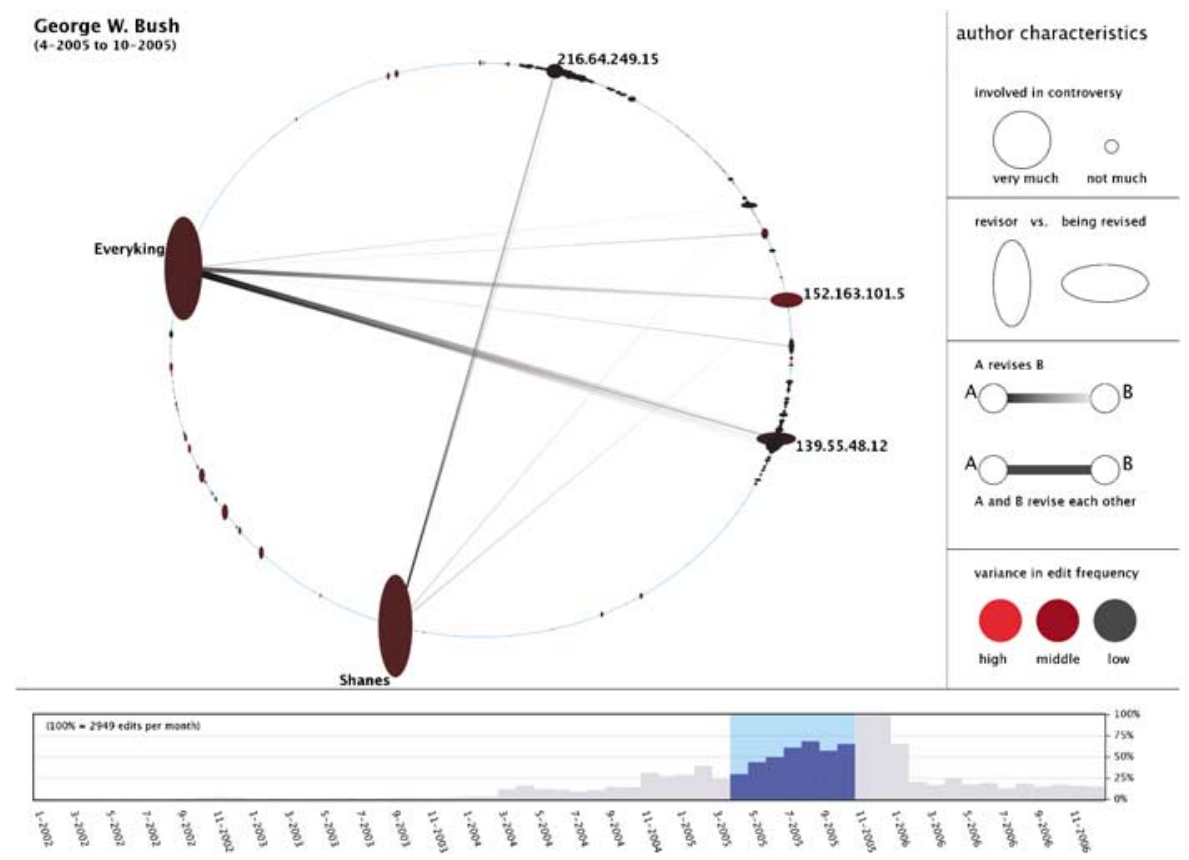

Figure 9 Revision network of the page George W. Bush in 2005. Two very busy revisors (Everyking and Shanes) opposed to numerous anonymous users -- all of them much less involved than the former two. It is likely that Everyking and Shanes play the role of 'watchdogs', defending the page against vandalism.

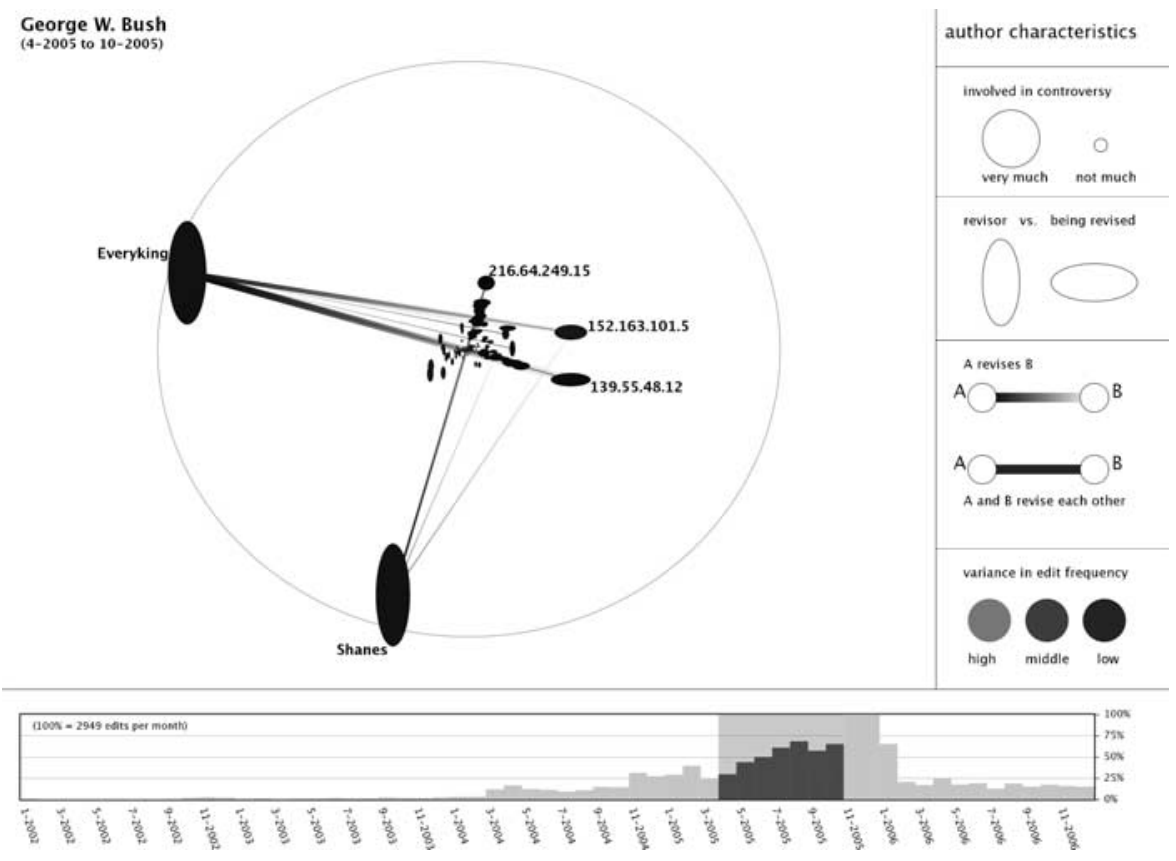

Figure 10 Same image as Figure 9 without the normalization of positions. The structure among the less-involved authors is hard to recognize. Note that the authors' involvement is also encoded in Figure 9 by their size.

very fast. To use terms that are easy to remember, the revised authors play the role of revolutionaries, the revisors the role of reactionaries. Note that these roles are to be understood relative to the content of a page: writing the page as desired by the revised would probably interchange the roles. 


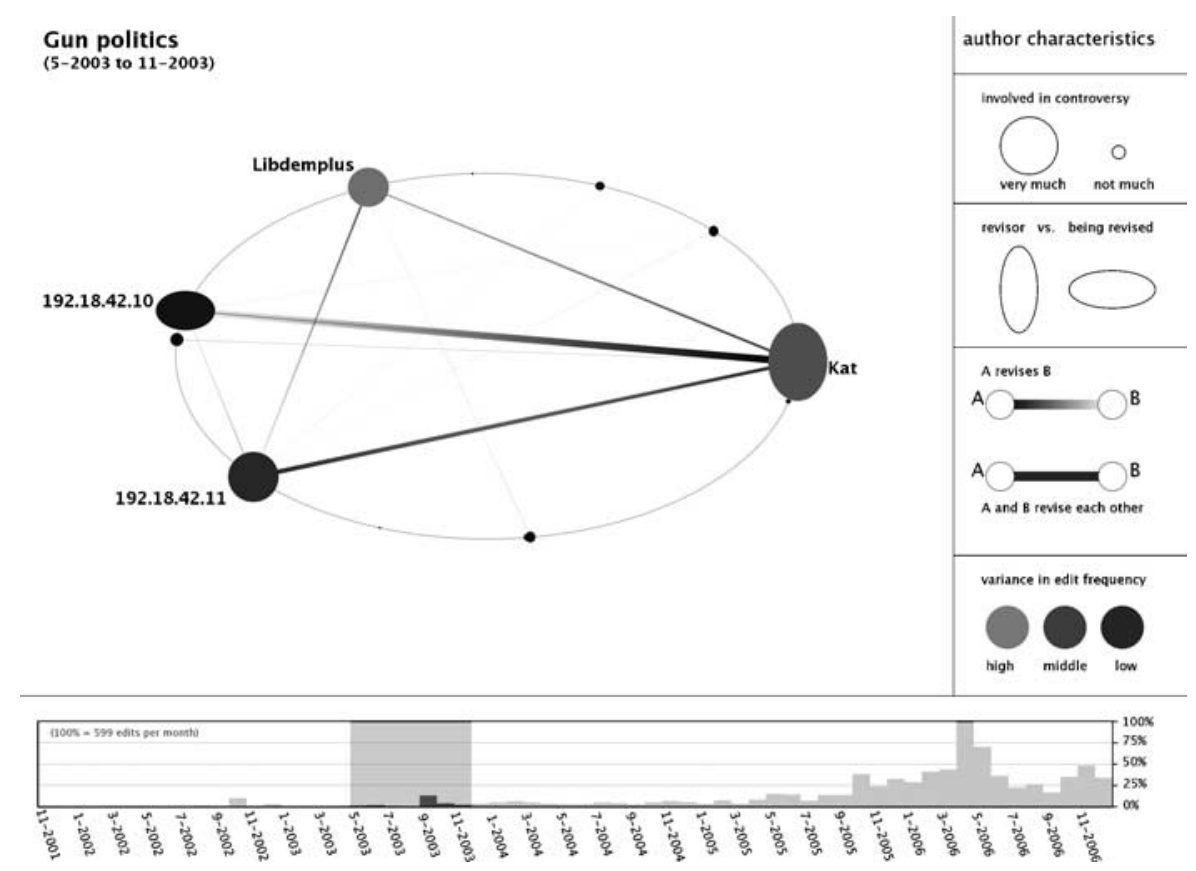

Figure 11 Filtering in time: a peak in the revision plot of Gun politics during 2003 has been caused by authors that vanish in the global image shown in Figure 4.

As an example consider Figure 4, where the anonymous author 24.12.208.181 is frequently revised - mostly by the revisors Yaf and Mmx1 (see Section 'Gun politics'). It is likely that authors keeping a page on their watch-list, as well as authors fighting vandalism also play the role of revisors (see Section 'Vandalism defense').

Direction of revision edges The edges show a dark-grey to light-grey gradient from the revising author to the revised author (compare the edge from Yaf to 24.12.208.181 in Figure 4). If an edge is almost symmetric it shows uniformly dark-grey. The information encoded by asymmetric edges is finer grained than that of the nodes' aspect ratio (as defined in Section 'Revisor vs being revised'): An author who is both, revisor and revised, appears as a circle, nevertheless, she may have asymmetric edges to some specific alters. The line thickness of an edge is chosen proportional to its weight and we show only the edges with the highest weights.

Steady vs unsteady participation One further indicator provides an important distinction between different author roles: there are authors that show sustained interest in editing a certain page and there are authors that perform a huge number of edits in a small time interval and loose their interest afterwards (or sometimes get blocked from editing Wikipedia). To assess this distinction we define a measure of how much does the weekly participation of an author vary. The decision 'one week' is in a certain sense arbitrary and exchangeable by longer or shorter intervals of time. However, we have chosen a week as this marks how people normally organize their work (an author being very active on weekends and inactive during the week will not be considered as unsteady).

Let a particular author and page be fixed and let $\left(e_{i}\right)_{i=1, \ldots, K}$ denote this author's number of edits on that page performed in week $i$. The sum $\mu=\sum_{i=1}^{K} e_{i} / K$ is the mean value (edits per week) and $\sigma^{2}=\sum_{i=1}^{K}\left(e_{i}-\mu\right)^{2} / K$ is the variance of the author's edit volume. However, variance is not yet an appropriate measure for the unsteadiness of a author, since authors with higher mean will normally have higher variance. This drawback can be overcome by considering the relative standard deviation $\sigma / \mu$. This makes sense since the edit volume is always positive (authors with no edits are not in the network). However, the normalization gives un-proportional weight to authors that have very small mean, for example, those that perform only one edit to the page. Since we are not interested in such peripheral authors, we will simply ignore them and apply the normalization only for those that exceed a certain minimum number of edits.

The relative standard deviation $\sigma / \mu$ is still not an appropriate measure for the unsteadiness, due to an observed characteristic of the input data: the variance of the aggregated number of edits (i.e., edits performed by all authors) can reach extremely high values (see, e.g., Figure 12), so that on those pages all (busy) authors will appear as highly unsteady. Since we are interested in differences between the authors (rather than absolute values), we subtract the 


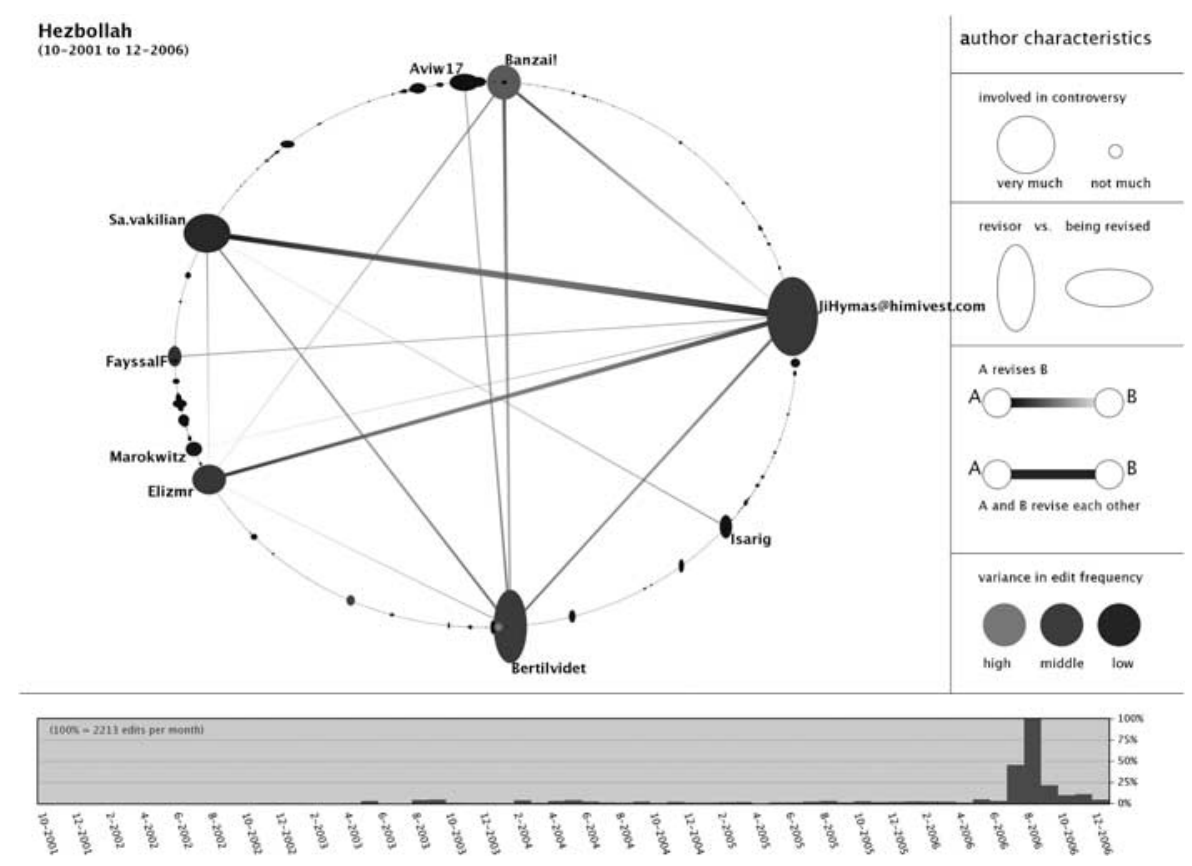

Figure 12 Page on Hezbollah has a very high variance in its edit volume. The strong peak in 2006 (2213 edits in August 2006 alone) is probably triggered by the 2006 Israel--Lebanon conflict (compare ${ }^{27}$ ).

minimum value of $\sigma / \mu$, so that the minimum becomes zero, and normalize so that the maximal value becomes one.

The node color of an author is black if this unsteadiness indicator is zero, that is, if the author showed sustained interest in the page. It becomes red if this indicator is one, that is, if the participation frequency is the most volatile. For instance, the anonymous user 24.12.208.181 in Figure 4 is slightly unsteady and Yafnot very unsteady (compare Section 'Gun politics').

Total number of edits per month The aggregated edit volume performed by all authors of the analyzed page (or set of pages) is visualized in a bar chart at the bottom of the image. This diagram provides the information about time periods when this page was a 'hot topic' (compare Section 'News triggered pages') and can also provide clues to restrict the revision network to interesting time intervals, see Section 'Restriction to time intervals'.

\section{Filtering}

Visualizing the complete revision network over the whole lifetime of the page gives an overview revealing the most important authors, the roles they play, and the other authors they confront. Next we describe how relevant sub-structures of the revision network can be determined.

Restriction to time intervals The edit volume diagram shown at the bottom of the images reveals time points when the page receives much interest. It is straightforward to restrict the revision network by including only revisions within a certain time interval. For instance, Figure 11 shows the revision network of Gun politics during a rise of interest in the earlier stages of the page. The dominant authors during that time are different from the dominant authors over the whole lifetime (shown in Figure 4). Restricting the network to specific time intervals also enables the analyst to examine the most recent development.

Restriction to relevant sub-networks A revision network often contains several ongoing controversies that are almost independent, that is, involving disjoint sets of authors. For instance, one controversy can be due to different opinions of the authors (see, e.g., Figure 4 and Section 'Gun politics') and another conflict can arise between vandals and vandalism repair (see, e.g., Figure 13). Since such controversies might overlap in time, it is in general not possible to separate them by restriction to time intervals as described in Section 'Restriction to time intervals'. Instead, an approach based on network clustering, which is described in the following, performs quite well in doing this task.

The goal of the network clustering is to put authors that strongly revise each other into the same cluster and authors that have only little interaction into different clusters. The sub-networks induced by the various clusters are then analyzed separately. In general, density-based graph clustering is a hard task (compare Gaertler ${ }^{28}$ ). We used a variant of spectral graph clustering heuristics proposed, for example, in Kannan et al. ${ }^{29}$ and McSherry. ${ }^{30}$ These 


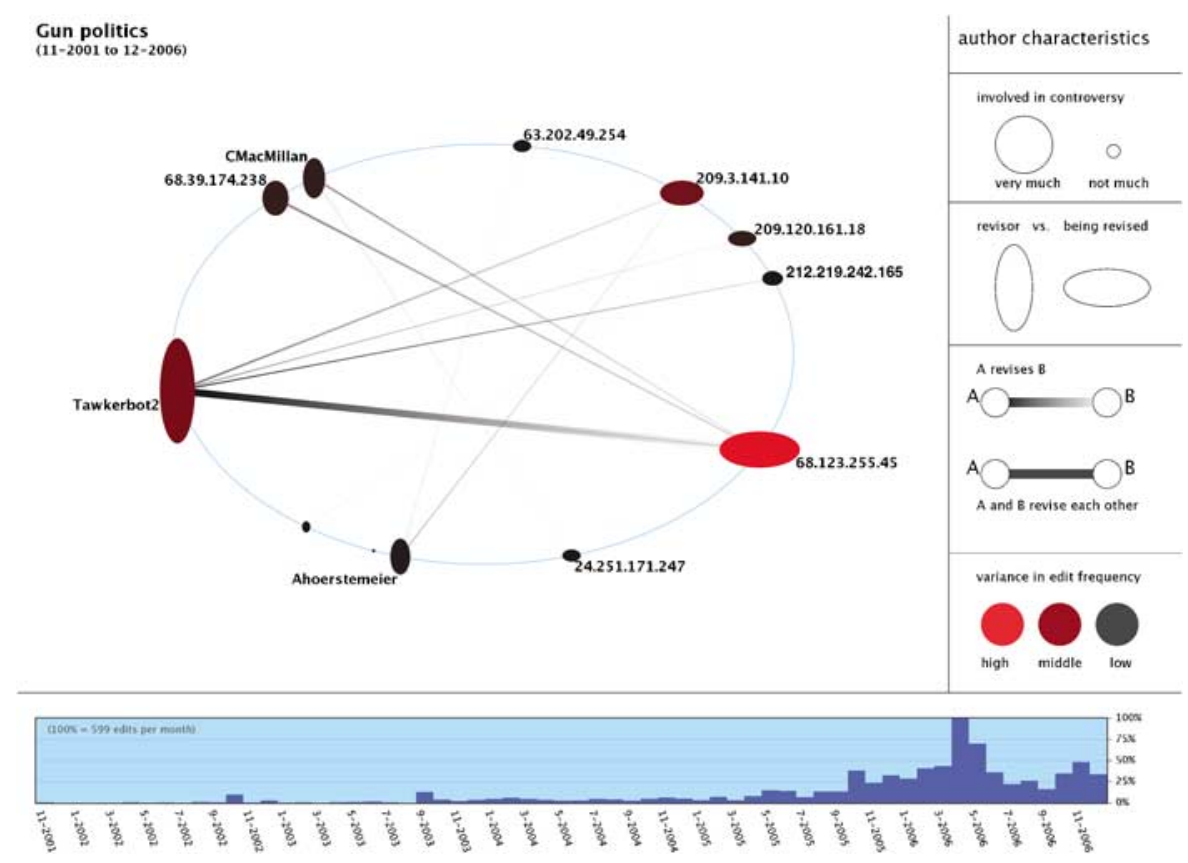

Figure 13 Network clustering reveals a relevant sub-network of the revision network of Gun politics. Another controversy cluster of larger aggregated edge weight is similar to Figure 4 and not shown separately. User Tawkerbot 2 is not a real author but a script for vandalism repair; its dominant opponents are anonymous users. It seems that this image shows revisions caused by vandalism, overlapping in time with the dispute over different opinions shown in Figure 4.

spectral heuristics are efficient, received much empirical and theoretical support (see Kannan et al. ${ }^{29}$ and McSherry $^{30}$ and references therein), and also performed quite well in the examples that we considered. Figure 13 shows a meaningful sub-network determined by network clustering.

\section{Examples of pages and patterns}

In this section, we describe a sample of illustrating findings on specific pages and some patterns that could repeatedly be observed.

\section{Gun politics}

The issue gun rights $v s$ gun control is a typical pro/con topic. Several Wikipedia pages, like Gun politics, Gun politics in the United States, etc. are related to this topic and have largely overlapping author communities. We took the union of the associated revision networks which are built together from 4609 revisions by 781 different authors. This network, which is shown in Figure 4, contains several interesting subnetworks that are extracted either by filtering in time (compare Section 'Restriction to time intervals') or by network clustering (compare Section 'Restriction to relevant sub-networks'). For space limitations we will describe only the global view in Figure 4.

The dominant confrontation in this network is clearly between Yaf and the anonymous user 24.12.208.181 (which we abbreviate in the following with 181). (Strictly spoken it is not clear whether the same IP implies the same person - however, looking at the sustained interest of 181 in gun politics makes us believe that this is the case.) Looking at Yaf's user-page - the user-page of a user uName can be accessed under

\section{http://en.Wikipedia.org/wiki/User:UName}

- makes it rather simple to guess that he/she advocates the freedom to carry guns. In contrast, looking at the contributions of 181 - all contributions of a user UName can be accessed under

\section{http://en.Wikipedia.org/wiki/Special:Contributions/ UName}

- makes it almost evident that he/she takes the opposite point of view. The author 181 shows a slightly unsteady edit behavior and is therefore drawn in dark-red in Figure 4. Indeed, 181 performed almost a hundred edits in Wikipedia - all of them between November 2005 and April 2006 and almost all to pages related to gun politics. Besides differences in opinion, another distinction between these two users is that Yaf is more a revisor and 181 more revised (see Section 'Revisor vs being revised'). The asymmetry of the edge between these two users is mostly due to a couple of very quick revisions (within less than 5 mins) where Yaf reverts edits made by 181 . 
Interestingly, some Wikipedia authors chose a username that itself expresses a certain orientation. For instance, the name GunsKill (also shown in Figure 4) already gives a indication that this author may advocate more gun control (looking at his/her contributions further supports this). It is remarkable that this user is - similarly as 181 more revised (mostly from Rhobite) than revisor.

While names like GunsKill indicate a certain opinion with respect to a specific topic, names like Yafnot indicate a negative feeling towards another Wikipedia user (Yaf in this case). Not surprisingly, Yafnot and Yaf are on opposite sides in Figure 4. Yafnot shows a very high variance in his/her edit behavior and is therefore drawn in red. Indeed, this author made only seven contributions to Wikipedia - all on April 2, 2006 in a period of less than $2 \mathrm{~h}$ and all to the page Gun politics in the United States. Author Yafnot is an example of a user that did not contribute much (only seven edits) but is quite a lot involved in controversy (among the nine most involved users in Figure 4).

Looking in detail at the sequence of edits of Gun politics in the United States on April 2, 2006, taking into account the positions of Yaf, Yafnot, and 181 in Figure 4, and considering the purposeful name of Yafnot, on could come to the hypothesis that Yafnot and 181 are the same person. Indeed, Yaf had the same idea, as the following quote (taken from the user talk page of Rhobite, archive nine ${ }^{31}$ ) indicates:

User 24.12.208.181 has apparently taken the user name Yafnot after your 2nd Level warning. He has continued to delete content of Gun politics in the United States. Thanks. Yaf 06:14, 2 April 2006 (UTC)

It is difficult to prove this hypothesis conclusively, without access to the log-files of the Wikipedia server. In any case, user Yafnot was blocked on April 2, 2006 (still less than $2 \mathrm{~h}$ after his/her first edit) by Rhobite for impersonation.

\section{Three-lateral conflicts}

While the page Gun politics is related to a typical pro/con topic, other topics can lead to more complicated controversy structures. The three most involved authors (Fossa, Perun, and Capriccio) of the page Kroatien (Croatia) in the German Wikipedia are mutually connected by strong revision edges and are therefore drawn in a triangle. (Note that, since the edge between Perun and Capriccio has smaller weight, these two users are drawn closer together, as it is claimed in Section 'Derivation of the scaling factor'.) The less involved authors are often connected to only one of the dominant three and are therefore drawn at the opposite position. The deeper reason for this three-polar conflict structure can only by found out by reading statements on user pages and user-talk pages. The history of Croatia is strongly connected to the wars following the end of the
Yugoslavian republic and disputes related to this page are often about the interpretation of historic events or over whether certain famous persons can be categorized as Croats or rather Yugoslavs. A rough qualitative evaluation of the related discussions suggests that some users favor more a Croatian point of view, others prefer a Serbian point of view, and yet others see themselves as Yugoslavs (or more generally as Europeans) and strongly oppose to nationalism of every direction. However, when analyzing the revision network related to Kroatien and reading the related discussions it became evident that our non-NLP approach reaches its limitations when applied to situations where we have to make such subtle distinctions in the disagreement relations. A promising direction for future work would be to merge the automatically generated revision network with an interaction network that has been constructed (manually or by a semi-automatic procedure) by a qualitative analysis of discussion pages, that is, interpreting discussion entries as supportive, neutral, or antagonistic with respect to some other author and adding this information to the revision network. Note that usually the number of entries on discussion pages is by far smaller than the number of revisions on the related Wikipedia pages. Thus, this procedure would still be applicable to high-interest pages, since the largest part of the work is done automatically.

\section{Vandalism defense}

A typical pattern emerges when analyzing the page George W. Bush. This page is the most edited in the English Wikipedia (more than 30,000 revisions by more than 10,000 authors), is a frequent target of vandalism, and was the first Wikipedia page that become protected (cf. Viégas et al. ${ }^{4}$ ).

The network visualization (see Figure 9) reveals two dominant users playing the role of revisors, which are opposed to a huge number of much less involved anonymous alters. User Shanes is a Wikipedia administrator and user Everyking a former administrator who had this status in 2005. A significant difference between the pages Gun politics (see Figure 4) and George W. Bush is that in the former the dominant authors confront dominant alters. It is likely that the users confronting Everyking and Shanes in Figure 9 are not really interested in writing a good article but rather want to vandalize the page. On the other hand, the dominant authors of Gun politics seem to care about its content, since they contributed a lot (although they have quite different ideas of what is a good Gun politics page).

Figure 10 shows the layout of the same network without the normalization of authors' position to an ellipse. The center of this drawing obviously gets too crowded and it is hard to recognize the positions of the unimportant actors.

\section{Obstinate vandals}

The revision network of some pages shows a structure that could be described by 'one user against the 


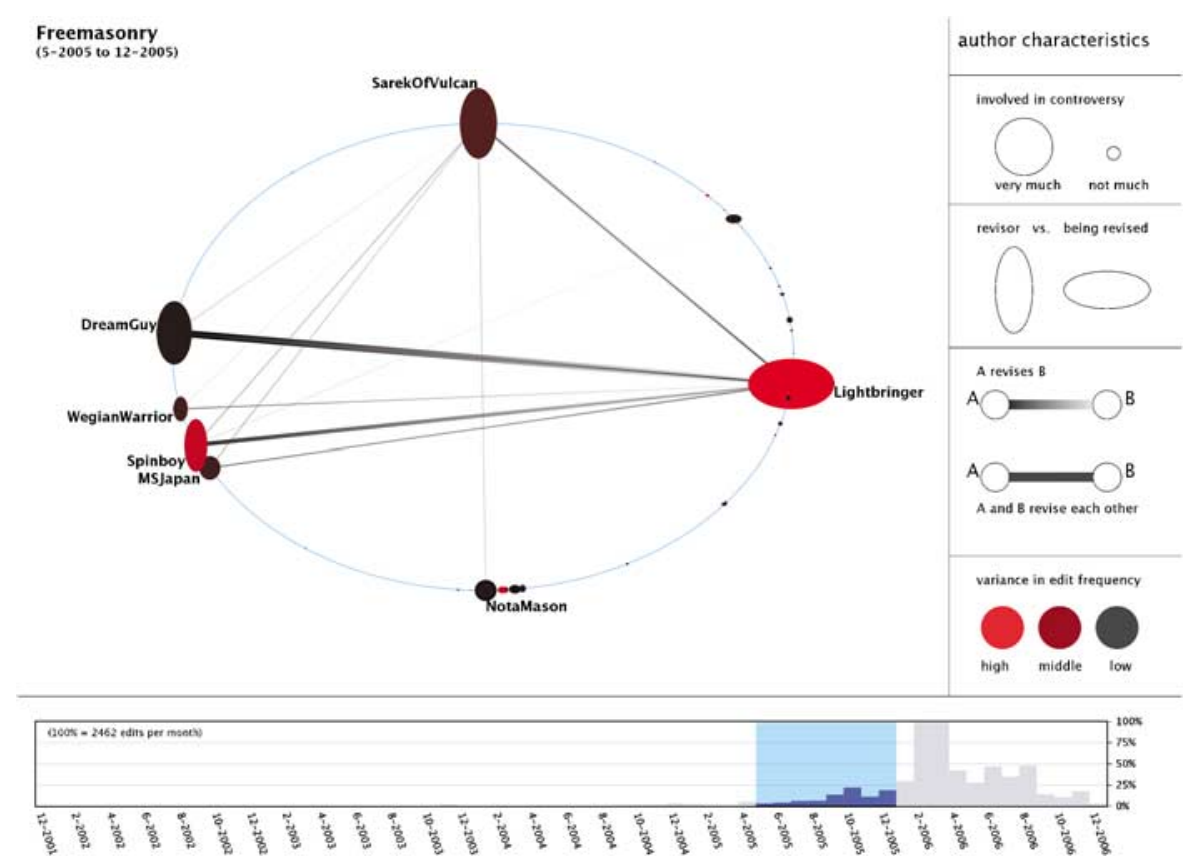

Figure 14 Revision network related to Freemasonry showing a certain author (Lightbringer) in opposition to nearly anybody else. Note that, in contrast to Shanes and Everyking in Figure 9, Lightbringer is more revised than revisor and, in addition, shows a very high variance in the edit frequency.

rest of the world.' Figure 14 shows the network related to Freemasonry around the end of 2005. The author Lightbringer is in opposition to most other authors, is more revised than revisor and shows a very unsteady edit behavior (drawn in red). Lightbringer's user page reveals that he/she is not only banned from Wikipedia but also tried to continue editing pages using various IPaddresses or different user names (so-called sock puppets).

On a first glance, it seems to be hard to distinguish between users who are fighting many vandals ('watchdogs', e.g., Shanes and Everyking in Figure 9) and users who are very active vandals opposed by many others (as Lightbringer in Figure 14), since both types of users stand rather on their own against a mass of other users. However, differences are the following two: first, vandals are more likely to be revised and vandal-fighters are more likely to be revisors. Second, active vandals that eventually became blocked have necessarily a high edit variance and therefore are shown in red color.

\section{News-triggered pages}

The edit history of some Wikipedia pages is strongly influenced by political events. An extremal example is the page on Hezbollah (see Figure 12). Although this page exists in Wikipedia since October 2001, it only became a hot topic during the 2006 Israel-Lebanon conflict and calmed down afterwards. We showed in Brandes and Lerner ${ }^{27}$ that the pages Hezbollah and 2006 Israel--Lebanon conflict indeed have a very high correlation in their edit frequencies over time. Furthermore, these correlation values could be used to establish relationships between pages with high edit variance.

\section{Conclusion}

Wikipedia makes it possible to assess the author community behind an article by providing the complete edit history of a page. However, the sheer number of edits and authors makes it hard to understand this data without automatic support.

The main contribution of our work lies in the proposed techniques for visual analysis of the revision network. Our drawings easily reveal the authors that are the most involved in controversy (taking the number of edits as a measure for user involvement would be insufficient as the example of Yafnot in Section 'Gun politics' shows). Furthermore, our network visualizations show who confronts whom and who plays which role.

Another contribution is that we identified some recurrent patterns of confrontation in the examples we considered: both Figure 4 and Figure 9 show a high asymmetry in the sense that users on one side of the conflict play the role of revisors and users on the other side are revised. However, the interpretation of the revisor $v s$ revised pattern can be quite different. In Figure 4 it seems to be caused by differences in opinion and in Figure 9 by vandalism.

One issue for future work is to determine more conclusively the difference between opinion-triggered and vandalism-triggered confrontation. Possibilities include 
to make use of log data about user blocking, statements on talk pages or user-talk pages, or contributions of an author to other pages. Another issue is to improve the construction of the revision network by taking into account whose text has been changed during a revision or by augmenting the revision network by relations determined from discussion pages, as this has been outlined in Section 'Three-lateral conflicts'. Note that the network visualization technique is totally independent from the network construction procedure, as long as the edges can be interpreted as disagreements or more generally as negative relations between actors. In particular, the visualization technique could be used to visualize controversies among users in other domains, for example, Usenet groups or blogs.

\section{Acknowledgements}

This research was supported by DFG under grant $\mathrm{Br}$ 2158/2-3.

\section{References}

1 Brandes U, Lerner J. Visual analysis of controversy in usergenerated encyclopedias. Proceedings of the IEEE Symposium on Visual Analytics Science and Technology (VAST'07) (Sacramento, CA), IEEE Computer Society: Chicago, 2007; 179-186 (Paper Chairs: John Dill and William Ribarsky).

2 Giles J. Internet encyclopaedias go head to head. Nature 2005; 438: 900-901.

3 Viégas FB, Wattenberg M, Dave K. Studying cooperation and conflict between authors with history flow visualizations. Proceedings of the SIGCHI Conference on Human Factors in Computing Systems (Vienna, Austria), ACM: New York, 2004; 575-582 (Conference Chairs: Elizabeth Dykstra-Erickson and Manfred Tscheligi)

4 Viégas FB, Wattenberg M, Kriss J, van Ham F. Talk before you type: Coordination in Wikipedia. Proceedings of 40th Annual Hawaii International Conference on System Sciences (HICSS'07), IEEE Computer Society: Chicago, 2007; 78a (Conference Chair: Ralph H. Sprague, Jr.).

5 Wikipedia. List of policies [WWW document]. http://en. Wikipedia.org/wiki/Wikipedia:List_of_policies (accessed 30 November 2007).

6 Kolbitsch J, Maurer H. The transformation of the Web: how emerging communities shape the information we consume. Journal of Universal Computer Science 2006; 12: 187-213.

7 Leuf B, Cunningham W. The Wiki Way. Addison-Wesley: Reading, MA, 2001.

8 Holloway T, Bozicevic M, Börner K. Analyzing and visualizing the semantic coverage of Wikipedia and its authors. Complexity 2007; 12: $30-40$.

9 Stvilia B, Twindale MB, Smith LC, Gasser L. Assessing information quality of a community-based encyclopedia. Proceedings of the International Conference on Information Quality (Cambridge, MA), 2005; 442-454 (unpublished) (Program Chairs: Michael Gertz, Michael Mielke, and Felix Naumann).

10 Voss J. Measuring Wikipedia. Proceedings of the International Conference of the International Society for Scientometrics and Informetrics (Stockholm, Sweden), 2005 (unpublished).

11 Gabrilovich E, Markovitch S. Overcoming the brittleness bottleneck using Wikipedia: Enhancing text categorization with encyclopedic knowledge. Proceedings of the 21st National Conference on Artificial Intelligence (Boston, Massachusetts), AAAI Press: New York, 2006 (Program Co-chairs: Yolanda Gil and Raymond J. Mooney).

12 Strube M, Ponzetto SP. WikiRelate! computing semantic relatedness using Wikipedia. Proceedings of the Twenty-First
National Conference on Artificial Intelligence (AAAI'06) (Boston, MA), AAAI: New York, 2006 (Program Co-chairs: Yolanda Gil, and Raymond J. Mooney).

13 Chen C, Ibekwe-SanJuan F, Sanjuan E, Weaver C. Visual analysis of conflicting opinions. IEEE Symposium on Visual Analytics'06 (Baltimore, MD), IEEE Computer Society: Chicago, 2006; 59-66 (Paper Chairs: Pak Chung Wong and Daniel Keim).

14 Glance N, Hurst M, Nigam K, Siegler M, Stockton R, Tomokiyo T. Deriving marketing intelligence from online discussion. Proceeding of the Eleventh ACM SIGKDD International Conference on Knowledge Discovery in Data Mining (Chicago, Illinois), ACM: New York, 2005; 419-428 (General Chairs: Robert Grossman, Program Chairs: Roberto Bayardo and Kristin Bennet).

$15 \mathrm{Liu} \mathrm{B}, \mathrm{Hu} \mathrm{M}$, Cheng J. Opinion observer: analyzing and comparing opini ons on the Web. Proceedings of the 14th International Conference on World Wide Web (Chiba, Japan), ACM: New York, 2005; 342-351 (Conference Chairs: Allan Ellis and Tatsuya Hagino).

16 Nigam K, Hurst M. Towards a robust metric of opinion. Proceedings of the AAAI Spring Symposium on Exploring Attitude and Affect in Text (Palo Alto, CA), 2004 (AAAI Technical Report SS-04-07) (Co-Chairs: Yan Qu, James G. Shanahan, and Janyce Wiebe).

17 Agrawal R, Rajagopalan S, Srikant R, Xu Y. Mining newsgroups using networks arising from social behavior. Proceedings of the 12th International Conference on World Wide Web (Budapest, Hungary), ACM: New York, 2003; 529-535 (Conference Chairs: Gusztáv Hencsey and Bebo White, Program Chairs: Yih-Farn Robin Chen, László Kovács, and Program Chair: Steve Lawrence).

18 Rosecrance RN. Bipolarity, multipolarity, and the future. Journal of Conflict Resolution 1966; 10: 314-327.

19 Kittur A, Suh B, Pendleton BA, Chi EH. He says, she says: conflict and coordination in Wikipedia. Proceedings of the SIGCHI Conference on Human Factors in Computing Systems (San Jose, CA), ACM: New York, 2007; 453-462 (General Chair: Mary Beth Rosson, Program Chair: David Gilmore).

20 Suh B, Chi EH, Pendleton BA, Kittur A. Us vs. them: understanding social dynamics in Wikipedia with revert graph visualizations. Proceedings of the IEEE Symposium on Visual Analytics Science and Technology (VAST'07) (Sacramento, CA), IEEE Computer Society: Chicago, 2007; 163-170 (Papers Chairs: John Dill and William Ribarsky).

21 Brandes U, Fleischer D, Lerner J. Summarizing dynamic bipolar conflict structures. IEEE Transactions on Visualization and Computer Graphics, special issue on Visual Analytics 2006; 12: 1486-1499.

22 Wikimedia. Downloads [WWW document]. http://download. wikimedia.org/ (accessed 30 November 2007).

23 SAX. Simple API for XML [WWW document]. http://www. saxproject.org/ (accessed 30 November 2007).

24 DOM. Document object model [WWW document]. http://www. w3.org/DOM/ (accessed 30 November 2007).

25 Kaufmann M, Wagner D. Drawing Graphs. Springer Verlag: New York, NY.2001

26 Golub GH, van Loan CF. Matrix Computations. John Hopkins University Press: Baltimore, MA.1996.

27 Brandes U, Lerner J. Revision and co-revision in Wikipedia. Proceedings of the International Workshop on Bridging the Gap Between Semantic Web and Web 2.0 at the 4th European Semantic Web Conference (ESWC'07) (Innsbruck, Austria), 2007; 85-96 (unpublished) (Workshop Chairs: Bettina Hoser and Andreas Hotho).

28 Gaertler M. Clustering. In: Brandes $U$ and Erlebach T (Eds). Network Analysis. Springer Verlag: New York, NY, 2005; 187-215.

29 Kannan R, Vempala S, Vetta A. On clusterings: good, bad and spectral. Journal of the ACM 2004; 51: 497-515.

30 McSherry F. Spectral partitioning of random graphs. Proceedings of the 42nd Annual IEEE Symposium on Foundations of Computer Science (FOCS'01) (Las Vegas, Nevada), IEEE Computer Society: Chicago, 2001; 529-537 (Program Chair: Moni Naor).

31 Wikipedia. Archived user talk page of [WWW document]. http:// en.Wikipedia.org/wiki/User_talk:Rhobite/Archive_9 (accessed 30 November 2007). 